\title{
Networking theoretical frames: the ReMath enterprise
}

\author{
Michèle Artigue, Université Paris Diderot - Paris 7 \\ Maria-Alessandra Mariotti, University of Siena
}

\begin{abstract}
In this article of the special issue devoted to the European project ReMath, we present and discuss the affordances of this project in terms of networking between theoretical frameworks. After clarifying the vision of theories and networking adopted in this project, we introduce the methodological constructs that have been developed and used in ReMath for performing the planned networking enterprise. We then present its outcomes, focusing on different facets of this networking activity: the identification of possible connections and complementarities between frameworks, the identification and elaboration of boundary objects between cultures, and the progressive building of a shared theoretical framework regarding semiotic representations. In the last part of this article, we review the whole networking enterprise from the perspective of research praxeology.
\end{abstract}

Key words: educational theories, theoretical networking, semiotic representations, digital technologies, cross-experimentations.

\section{Introduction}

Recently, in the field of mathematics education, increasing attention has been paid to theoretical diversity and attempts have been made aimed at overcoming what can be perceived as a state of fragmentation of the field. This increasing attention is attested, for instance, by the existence of a working group devoted to theoretical issues focusing on the comparison and networking between theoretical frameworks at the CERME Conferences since 2005 , by the publication of special issues of journals devoted this question such as the ZDM issues published in 2006 and 2008, (ZDM, vol. 38(1) and 40(2)), by the organization of a specific research forum at PME34 (Bikhner-Ahsbahs \& al., 2010), and by the publication in 2010 of the volume entitled Theories in Mathematics Education (Sriraman \& English, 2010) whose part XVI is a series of chapters devoted to the theme. As explained in the introduction of this special issue, contributing to this collective enterprise was an essential ambition of ReMath, while focusing on one particular area of research: teaching and learning in digital environments, and on a particular dimension of research in that area: the semiotic dimension. The first ReMath Deliverable (Artigue, 2006) indeed presented the project in the following way:

At the origin of ReMath lies the fact that the huge efforts which have been performed in most European countries to improve mathematics education through the development and use of Interactive Learning Environments (ILEs), have had a limited impact on the reality of school practices up to now. [...] For the ReMath partners, part of the difficulty comes from the fragmented character of the theoretical frames which have been developed in order to approach learning and teaching processes in mathematics education. Part of the difficulty also comes from the insufficient attention that research tends to pay to contextual issues, both for design and use, and especially to the distance separating the experimental contexts where the learning potential of ILEs is generally evidenced from the context of ordinary classrooms.

Thus the ReMath project aims at looking for integrating perspectives in terms of theoretical frameworks, and made the choice to closely link their construction to the development of some specific ILEs and to experimentations of these carried out in realistic educational contexts. (p. 5) 
Therefore, with respect to theoretical networking, high ambition was given to the project as shown by the scale proposed by Prediger, Bikner-Ahsbahs \& Arzarello (2008) and reproduced in figure 1. The plan was not only to contrast and establish connections between theories but also to make progress towards some form of local integration, that is to say towards the development of new theoretical constructions by integrating elements of different theoretical perspectives. A major goal was discriminating networking strategies, in terms of their degree of integration.

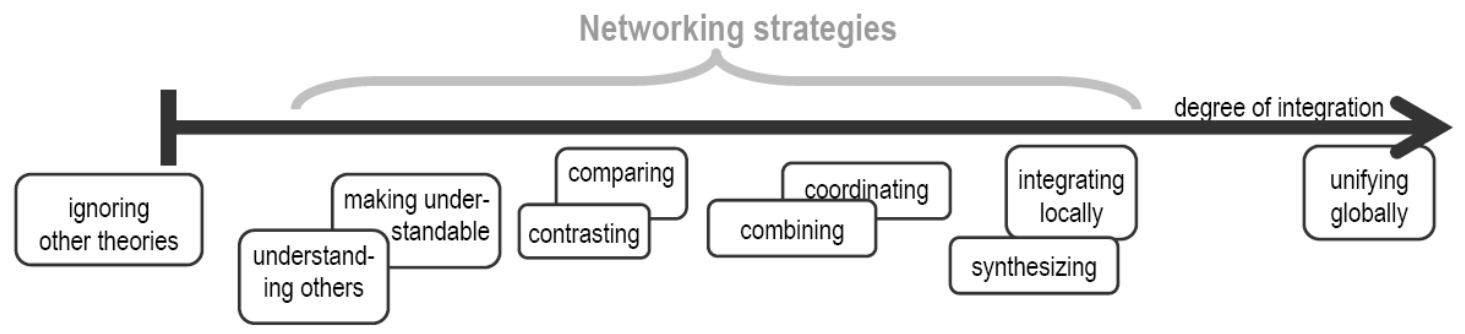

Figure 1 Networking strategies (Prediger \& al., 2008, p. 170)

In this article, we present, analyze and discuss the research developed in ReMath regarding the networking of theoretical frames and we try to make clear both the advances it has permitted and its limitations. This project has led not only to practical results in terms of networking but also to methodological advances. For this reason, methodological issues are given an important place in this paper. In the first section, we present ReMath's vision of theories and theoretical networking, situating it with respect to other possible perspectives. In the second section, we show how this vision has impacted the networking activities developed along the four years of the project, paying particular attention to the constructs and methodological tools which made this vision operational. In the third section, we come to the results obtained in terms of networking, and in the fourth and last section we review the whole enterprise with respect to the idea of research praxeology (Artigue, Bosch \& Gascón, 2011).

\section{Delineating a particular vision of theories and theoretical networking}

As shown by the literature, in mathematics education there co-exist different visions of theories. For instance, Niss (2007) defines a theory in a static view:

as a system of concepts and claims with certain properties, namely:

- A theory consists of an organized network of concepts and claims about some extensive domain, or a class of domains, consisting of objects, processes, situations and phenomena.

- In a theory, the concepts are linked in a connected hierarchy, in which a certain set of concepts, taken to be basic, are used as building blocks in the formation of other concepts.

- In a theory, the claims are either basic hypotheses, assumptions or axioms, taken as fundamental, or statements obtained from the fundamental statements by means of formal or material derivation". (p. 308)

In the ZDM special issue mentioned above, Radford (2008), for his part, defines a theory as a way of producing understandings and ways of action based on:

- A system, $P$, of basic principles, which includes implicit views, and explicit statements that delineate the frontier of what will be the universe of discourse and the approached research perspective.

- A methodology, $M$, which includes techniques of data collection and data interpretation as supported by $P$. 
- A set, $Q$, of paradigmatic research questions (templates or schemas that generate specific questions as new interpretations arise or as the principles are deepened, expanded or modified)." (p. 320)

Inspired by the previous work carried out by the same partners within the European Research Team TELMA (TELMA ERT, 2006), (Artigue, 2009a), in ReMath we considered theories as structured systems emerging from research practice and conditioning all facets of these practices: the articulation of problems and questions, the methodologies used for addressing these, the data collected and their interpretation, what are identified as research results, and how the connections between research and practice are envisaged. We have also considered theories as dynamic entities impacted by the ways researchers use and accommodate them, as well as by the evolution of educational cultures where they live and develop. This structured but dynamic vision of theories combines characteristics of the two types presented above. It is in line with the vision developed by Prediger, Bikner-Ahsbahs, \& Arzarello (2008) but it can also be modelled within the anthropological approach of didactics developed by Chevallard as described by Artigue, Bosch, \& Gascón (2011), where theories are conceived as ingredients of research praxeologies. We come back to this point in the last part of this article.

In line with this vision, we considered that substantial progress in theoretical networking required researchers to elaborate methodologies making it possible to access theories on the base of their relationship with research practices, thus paying particular attention to their operational dimension. Such methodologies should make it possible to connect theoretical frameworks but prevent adaptations becoming distortions in their desire to establish connections.

The TELMA experience had also shown us that in the research literature, theories are often invoked but the exact way they impact research practices is often not so clear. In many texts, theories seem to be used at a general and metaphorical level more than at an operational level. Moreover, this experience had shown us that most familiar theories and constructs tend to naturalize in the researchers' discourse and practice. We thus had the conviction that networking methodologies should make the invisible and tacit, visible and explicit, and address the obstacle of naturalization.

Such a vision of networking, once again, is in line with that expressed by different authors (cf. for instance (Bikner-Ahsbahs \& al., 2010; Radford, 2008), but the context of ReMath offered us the possibility of pushing the research implications of this vision further than is usually the case in the literature, creating a space where different theoretical cultures could really interact in the long term, building meta-languages and methodologies for efficiently supporting this interaction. In fact, ReMath offered us the possibility of creating what Radford (2008) referring to Lotman calls a semiosphere, that is: "an uneven multi-cultural space of meaningmaking processes and understandings generated by individuals as they come to know and interact with each other" (p. 318), which he claims to be a condition for developing productive theoretical networking.

Before entering into the details of the work and achievements of ReMath, we find it necessary to briefly situate the initial state of this multi-cultural space, focusing on its theoretical diversity. As has been explained in the introduction of this special issue, ReMath organized the collaboration of six different teams from four different countries: France, Greece, Italy and the UK. These teams lived in quite different educational contexts; for instance, in countries such as France and the UK, institutional constraints are much stronger than in Greece and in Italy. All teams had expertise in the technological domain (both in the design of technological artefacts and in their educational use). However, their theoretical cultures and references were quite different. 
They included home-grown theories such as the Theory of Didactical Situations (TDS) (Brousseau, 1997) or the Anthropological Theory of Didactics (ATD) (Chevallard, 1992; Chevallard \& Sensevy, 2013), and theories which have emerged and developed outside the field of mathematics education but have been adapted through their shared use inside the field of mathematics education, such as Activity Theory (Leont'ev, 1978; Engeström, 1999), or Social Semiotics (Halliday, 1978). They also included theories of different size and scope, and especially some theories more focused on semiotics such as Duval's semiotics (1995) or technology such as the Instrumental approach (Lagrange, 2000; Artigue, 2002) due to the specific aim of the project. Some theories resulted themselves from theoretical networking. The Instrumental approach for instance is an original construction resulting from the local integration of constructs and perspectives from Rabardel's Ergonomic approach (Rabardel, 1995) and the Anthropological theory of didactics (Artigue, 2009b). The Theory of Semiotic Mediation (TSM) (Bartolini \& Mariotti, 2008) is an original construction that elaborates on the notion of mediation introduced by Vygotsky (1978) and describes how the teacher can exploit the use of an artefact, managing different didactical situations to make expected semiotic process happen. Beyond that, all teams involved had already some practical experience of networking when ReMath started. The two French teams for instance were used to combining the use of the Theory of Didactical Situations and the Anthropological Theory of Didactics, two theories which have emerged and developed in their culture and whose connections have been systematically studied by the French community. The Italian teams were used to combining Activity Theory with Peircean semiotics (Pierce, 1931/1958); the UK team included both constructionist (Harel \& Papert, 1991), (Kafay \& Resnick, 1996) and social semiotic experts working together in the project. Moreover, most teams had incorporated the distinction made by between artefact and instrument, and the associated idea of instrumental genesis ${ }^{1}$ (Vérillon \& Rabardel, 1995), as well some notions of Duval's semiotics (Duval, 1995).

The Figure 2 below synthesizes the main elements of the initial theoretical landscape, those evoked in this article, both in terms of objects and connections, and situates each ReMath team within it. Connections are symbolized with arrows, bold arrows corresponding to well established connections that had already resulted in some local integration.

Thus the theoretical background of each team already resulted at this initial stage from some formal or informal networking, but these networking activities were internal to a given culture. The ambition of ReMath was to make networking progress beyond these cultural frontiers. With this perspective in mind, it is interesting to note that some boundary objects ${ }^{2}$ (Lave \& Wenger, 1991) already existed in that initial stage, in the form of common theoretical references, for instance the common reference mentioned above to (Vérillon \& Rabardel, 1995), or at a lesser extent Duval's semiotic approach in terms of registers of representation (Duval, 1995). As is the case for boundary objects between communities of practice, the ways these theoretical approaches were appropriated and used by the different teams had no reason to be similar but the existence of such objects was certainly promising in terms of networking.

\footnotetext{
${ }^{1}$ The artefact, here the DDA or some part of it, is distinguished from the instrument it becomes for someone through the elaboration and appropriation of schemes of use and instrumented action. The corresponding process is called instrumental genesis, and it affects both the artefact (instrumentalization process) and the user (instrumentation process).

${ }^{2}$ Star and Griesemer (1989) define boundary objects as "objects which are both plastic enough to adapt to local needs and the constraints of several parties employing them, yet robust enough to maintain a common identity across sites" (p. 393).
} 
At a more local level, other theoretical references were shared. This was especially the case regarding algebra, a domain embedded in several digital artefacts considered in ReMath, with the shared distinctions made between "structural" and "process" conceptions of algebraic objects, between "meaning" and "denotation" or algebraic expressions, just to mention a few examples.

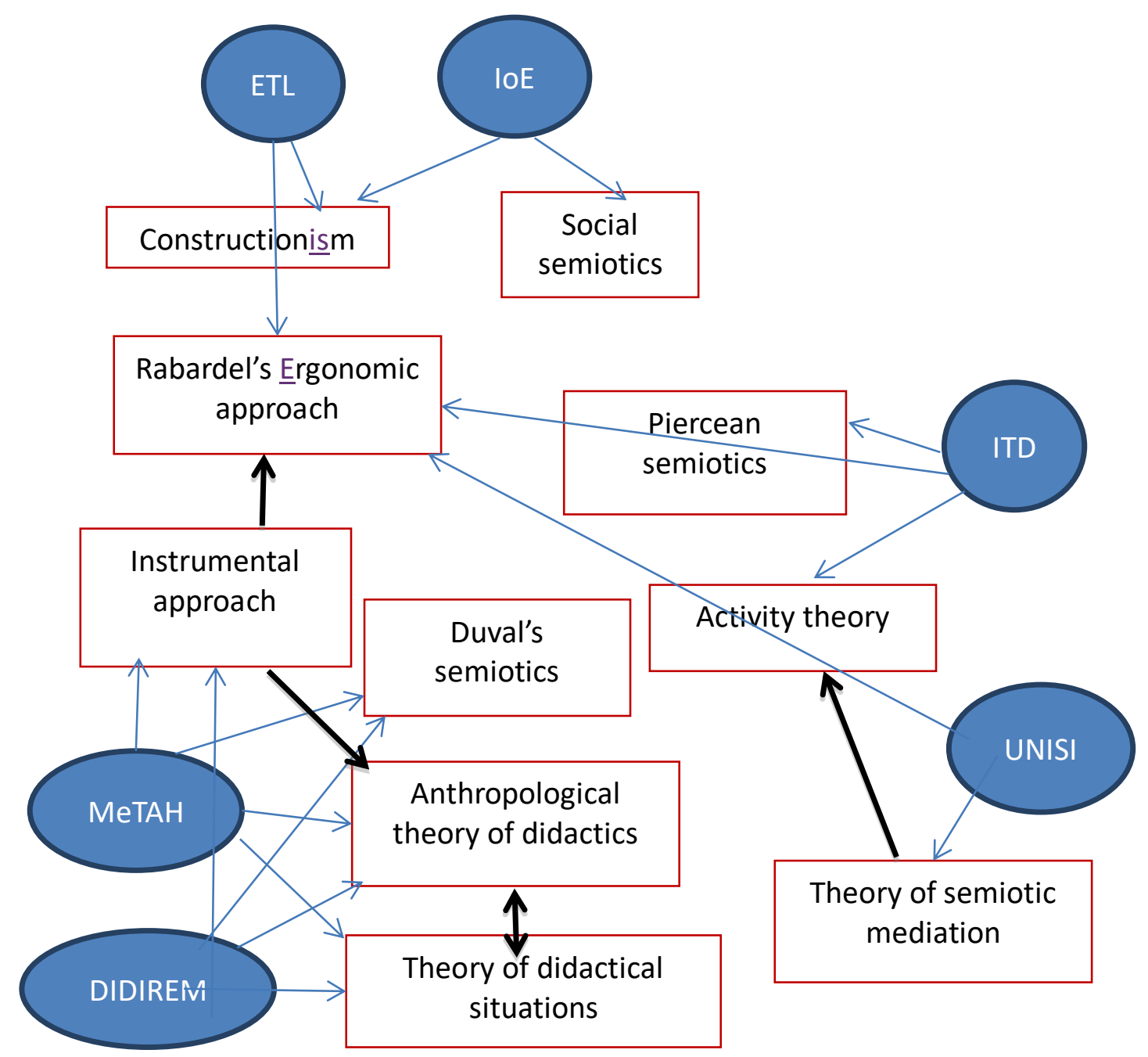

Figure 2: The initial theoretical landscape (In the balloons are the acronyms of the six partners teams of the ReMath project $)^{3}$

\section{Insightful examples for illustrating this vision}

Within TELMA the ReMath teams had already built the first elements of the semiosphere mentioned above. Among these elements there were the notion of didactical functionality and

\footnotetext{
${ }^{3}$ ETL is a Greek team, IoE a UK team, ITD and UNISI two Italian teams, MeTAH and DIDIREM (now LDAR) two French teams.
} 
the meta-language of concerns that we present in 3.1, showing how, reworked, they contributed to the ReMath enterprise. The idea of cross-experiment also emerged in TELMA, but then in ReMath it was developed and consolidated as a networking methodology; we present and discuss it in 3.2. Finally, in ReMath, theoretical frames were not only involved in the identification of the didactical functionalities of existing digital artefacts; they were also involved in the design and development of digital artefacts. Integrating this new dimension into theoretical networking required some specific methodological constructions. We focus in 3.3. on one of these, that of epistemological profile for a given dynamic digital artefact (DDA).

\subsection{At a conceptual level, the notion of didactical functionality and the meta-language of concerns}

As mentioned above, the notion of didactical functionality and the meta-language of concerns had already emerged in TELMA ((TELMA ERT, 2006). However, TELMA had focused not so much on design but mainly on the analysis of uses of existing DDAs, while in ReMath the design of both DDAs and scenarios of use became an essential dimension. Moreover, unlike in ReMath, the work carried out in TELMA was comparative and did not have integrative aims. So in ReMath the notion of didactical functionality and the meta-language of concerns were combined and used in an original way for establishing the foundations of what was called an Integrated Theoretical Framework (ITF), which was progressively refined along the four years of the project.

More precisely, the notion of didactical functionality, introduced for supporting the operational vision of theories underlying the project presented in section 2, was defined in the following way:

Given an ICT tool, it is possible to identify its didactical functionalities: with didactical functionalities we mean those properties (or characteristics) of a given ICT, and their modalities of employment, which may favour or enhance teaching/learning processes according to a specific educational aim".

"The three key elements of the definition of the didactical functionality of an ICT tool are then:

1. a set of features / characteristics of the tool

2. an educational aim

3. modalities of using the tool in a teaching/learning process referred to the chosen educational aim.

Designers of educational artefacts have didactical functionalities in mind and these inform design, but, as evidenced by research, users may also have a creative role and contribute to the didactical functionalities of artefacts through the modalities of use they envisage and implement. For both designers and users the didactical functionalities identified are dependent on their theoretical culture, thus didactical functionalities can be used as windows onto the operational dimension of theories. Hence comes the importance attached to didactical functionalities in the ReMath methodology.

The meta-language of concerns, for its part, can be seen as a consequence of the advanced hypothesis that different teams involved in a common project, even if living in different contexts and cultures and relying on different theoretical frameworks, more or less face similar problems and ambitions, and develop similar sensitivities. The notion of concern tries to capture these commonalities taken as a possible basis for communication and integration of perspectives. However, it was also hypothesized that not all common concerns are given the same emphasis by the researchers, and that even when they seem to be given a similar importance, they are not necessarily expressed or dealt with in the same way, that is with the same conceptual tools. Understanding these differences and their possible impact on design decisions was considered crucial for the success of the networking enterprise. 
Merging the frame of didactical functionalities with the meta-language of concerns while adopting a design perspective produced the core of a framing tool aimed at supporting and developing the process of networking, and the progressive elaboration of what we called the ITF. The first step consisted in providing a description of the specific theoretical positions expressed in a common language and this was achieved by setting up a structured questionnaire that each Team was asked to fill out (cf. Appendix 1). Considering the specific focus of ReMath on representations and contexts, the presentation of concerns was organized around these two elements for each of the three dimensions attached to the notion of didactical functionality. In order to limit the complexity of the tool, the analysis of representations was restricted to that of external representations and these were considered according to two dimensions:

- representation of objects;

- representation of interaction.

For similar reasons, for the analysis of context we also identified only two categories, that of:

- a local or situational context;

- a global or institutional and cultural context.

This way the ReMath teams tried to elucidate the role played, both explicitly and implicitly, by theoretical frameworks, to identify potential interesting connections and complementarities, and also divergences, potential misunderstandings and conflicts they needed to be aware of in order to start their integrative work. According to the objective and research plan of the project such work was expected to rely on local experimentations that would provide common and comparable results. This opened the question of designing an experimental methodology that could support the achievement of such an ambitious objective. For that purpose, as already mentioned, we were inspired by our work within TELMA, which had shown the interest of a methodology of cross-experimentation where each team was asked to develop an experiment with a DDA developed by another team, in another educational context and under other theoretical perspectives.

\subsection{At a methodological level, the cross-experiments and the attached guideline structure}

As we had learned from the TELMA experience, one of the main difficulties that researchers meet in reporting on a research study is that of making explicit some of the main assumptions of their work, but also to relate them to the results that are to be reported. Taking into account the support given by the ITF described above, the effort was concentrated on the design of a methodological tool conceived as a support for making crucial theoretical elements explicit, but also for making them explicit within a common frame that would allow the teams to share their results. This second objective, which was not part of the TELMA legacy, is to be related to the intention of developing a networking methodology. Actually, the common frame that was designed became the methodological element on which we based the assumption that we would be able not only to compare our local results, but also to integrate them into common answers to the main questions posed in our research project.

\section{Cross-experimentation revisited}

Within ReMath, we thus decided to retain the cross-experimentation methodological frame developped in TELMA whose principal characteristics consisted in:

- the design and implementation by each research team of a teaching experiment making use of a DDA developed by another team;

- the construction of a common set of guidelines expressing questions to be answered by 
each designing and experimenting team.

The first characteristic aimed at creating a perturbation in the research methodology, obliging researchers to face unfamiliar artefacts implementing other perspectives on the semiotic affordances of technology; such a perturbation was expected to foster explicitation of theoretical assumptions, in order to overcome the naturalization obstacle mentioned in section 2. The second characteristic aimed at fostering the process of cross-team communication. In the case of the ReMath project the choice of using again a cross experimentation methodology had a more ambitious objective. It was not just that of comparing different approaches, we wanted to make these approaches interact in order to achieve results that could be communicated in a shared form. The contraints created by the guidelines were expected to support the fact that the results emerging from the different teaching experimentations could enrich each other's: such results should contribute differently but in a compatible way to the main issue addressed by our Project, not only validating the use of each DDA as a didactic tool but also deepening our understanding of meaning-making through representing with digital media (ReMath Project Proposal part B, p. 59).

In spite of the common focus on digital representation and the shared hypothesis on the proactive role that such a type of representation may have in mathematics education, we immediately realized the great diversity of our positions and overall the consequent danger of producing fragmented pieces of results, pieces that though interesting in themselves might not be fruitfully compared and integrated into a common view. Thus, since the very beginning, one of our main concerns was preparing the terrain to foster the possibility of comparing and integrating the different results coming from different teaching experiments. The problem of articulating the local level of each teaching experiment and the global level of the cross experimentation was raised and discussed among the different teams, with the objective of sharing the delicate work of framing without constraining the different research studies. We present in what follows how this work progressively developed and the different methodological artefacts that accompanied this development, relying more especially on the ReMath Deliverables 11 and 13 devoted to the cross-experimentation (Mariotti, Maracci, \& Sabena, 2007; Mariotti \& Maracci, 2007).

\section{The conception of the guidelines}

A preliminary work pointed out the need of making explicit our common basic assumptions and of developing a shared language for dealing with representations. Such preliminary work led us to the definition of a first methodological tool, which was called Guidelines for the Teaching Experiments. This methodological tool aimed at framing the design of the different teaching experiments, in particular the research questions addressed in each teaching experiment in order to reach uniformity without losing the richness of diversity. The construction of the Guidelines also aimed at producing an organizing frame for collecting information on the planned local experiments. It consisted in two forms that each team was asked to fill out: a Protocol of experimentation and a Profile of experimentation.

- The Protocol had the goal of describing the main characteristics of the teaching experiment making explicit the Research Questions as they were addressed and framed in the specific theoretical framework chosen by the research team.

- The Profile of experimentation maintained the structure of the ITF: for each dimension of didactical functionalities, teams were asked to grade the associated concerns from 0 to 5 , reflecting the level of priority given in the design of the experiment.

A first version of the guidelines was proposed to the research teams and the researchers filled out the requested forms. 
The analysis of the Protocols and Profiles filled out showed the complexity of using the language of concerns. The most evident result was that each team graded as a high level almost every concern. Thanks to some emendation of the guidelines, this difficulty could be overcome, nevertheless it highlighted, as a key point, the complexity of making explicit the focus of one's own research, neglecting aspects that still appear crucial though not directly addressed. The difficulties met made it clear for us that networking between theoretical frameworks could help researchers to become aware of the fact that research emerges as an optimization process in a complex system, but that we hardly have complete awareness of such complexity and of the part of it we more specifically address.

Generally speaking, the analysis of a first version of the Protocols and Profiles showed the complexity of the process of making explicit one's own main assumptions through the frame of concerns. In particular, a comparison between the declared concerns and the research questions showed that not all the teams succeeded in formulating research questions clearly related to their indicated top concerns. In spite of the effort made in filling out the guidelines, the links between research questions, concerns and theoretical framework still remained mostly implicit. During one of the meetings of the Project, an animated discussion took place on the analysis of the first Protocols and Profiles produced by the different Teams; the following points emerged as shared among the different research Teams:

- sharing a certain concern should allow us to integrate local results;

- however, this may not be enough, since even a shared concern might be too general to allow integration.

The main result of our reflection was the awareness of the crucial role played by the formulation of research questions in connecting the particular focus of the local investigation and the general perspective of the common project: on one hand, research questions led the local experimental designs, which were intended to answer them, on the other hand, research questions should act as linking tools between the different experimentations in order to produce general answers to the main research objective of the ReMath project. A possible solution was hence envisaged in finding a way to articulate a shared concern into shared research questions. Research questions were then conceived as pivotal elements to pass from the general level of concerns to the specific level of experimental designs. For the purpose of each teaching experiment, the relationship between concerns and research questions needed therefore to be made explicit in a clear and precise way. Furthermore, for the purpose of integrating and comparing the different results, a common language was needed to articulate such research questions. The networking process was then oriented to set up this new base on which a new version of the Guidelines could be produced.

This phase of preparation was quite long and animated by a rich discussion that lasted beyond the project meeting. Two important decisions were thus taken. The first one was to agree on some main assumptions that would converge into a shared idea of representation and constitute what was called a Minimal Theoretical Framework on representation. The second one consisted in the articulation of a Common Research Question (CRQ in the following) that each team was asked to accept and rephrase according to its specific sensitivities and theoretical frameworks.

The Minimal Theoretical Framework was made of the following three assumptions presented as a skeleton of an implicitly shared theoretical framework concerning representation:

1. No direct access to mathematical objects is possible, rather mathematical meanings are represented through language, formal mathematical notations, informal idiosyncratic 
representations;

2. Representations play a fundamental role in the "generation" of mathematical meanings, and this role is assumed to be crucial in the teaching/learning of mathematics;

3. Digital artefacts can provide representations of mathematical objects with a clear potential of generating mathematical meanings;" (Mariotti \& Maracci, 2007, p. 10)

Such common assumption was made explicit together with a description of the idea of representation in the following terms:

A representation can be seen as a relationship between:

- $\quad$ a representing, i.e. something with a perceivable nature, accessible to one's senses, and

- a represented, i.e. something which is not accessible to one's senses but which is considered as existing in some sense (e.g. an idea, a concept, a process...).

Such relationship does not exist in itself, it is such only from someone's perspective. That is to say that a representation is such only if there is someone who recognizes a relation between something perceivable - a representing - and a corresponding represented. (ibid. p. 10)

It was added that this perspective taken on representation entails that individuals can share representations only through sharing the perception of the perceivable representing. And it was also acknowledged that it may happen that in spite of common perception of the representing, individuals fail to share the represented.

The Minimal Theoretical Framework was made of the three assumptions presented as a skeleton of an implicitly shared theoretical framework concerning representation, and a Common Research Question was articulated. This CRQ was the following:

How can the representations identifiable in the DDAs be put in relationship with the achievement of specific educational goals?

ReMath teams were asked to reformulate such a CRQ for each teaching experiment carried out and according to the specific theoretical framework assumed to frame their own investigation. The key idea was that on the one hand the different theoretical frameworks should provide means to refine the CRQ, articulating the general common issue according to different theoretical tools, but preserving its essence as expressed in the Minimal Theoretical Framework. Such reformulation should also be accompanied by comments where each team was asked to explain the key elements of its specific theoretical framework mobilized in it. Beyond that, the ReMath teams were also offered the possibility of articulating specific research questions, and they were of course also expected to link these specific questions to their key concerns and theoretical frameworks. On the basis of the new shared frame, the Guidelines were completely reorganized into a new document consisting of two main sections. The first section was devoted to framing the formulation of the research questions according to the CRQ and the second was devoted to supporting the research Teams to make explicit the relationship between the top research concerns and the reformulation of CRQ. Thus the initial Profile of experiment was refined for better structuring the information, supporting the Teams in their effort of making clear the various elements they focussed on. This refinement gave birth to a specific form, called Research Profile of the Teaching Experiment. Considering the limited discriminating power of the language of concerns as initially used, such Research Profile was meant to make it possible to isolate research concerns from other more general concerns that could inspire the teaching experiments at large. The teams agreed that, when filling out the Research profile, they should express only their specific research concerns. Consistently, each team should formulate research questions related to its own top research concerns; the link among research questions and related concerns must be made clear as well.

$\underline{\text { Some examples of reformulation }}$ 
In this section, we present and comment on some examples of reformulations of the CRQ. The first reformulation concerns the DDA Alnuset and is provided by the ITD team who were also the designer of this DDA.

Team A (ITD):

How can the algebraic representations mediated by Alnuset be acted on? Which kind of relationship do these algebraic representations establish with the referential algebraic objects and phenomena?

Can an integrated use of these algebraic representations be effective to mediate the development of educational practices based on integration between an algebra of quantities and an algebra of operations?

Are these educational practices mediated by Alnuset useful to construct meanings for the use of letters in algebra and to understand what algebraic expressions denote?

Are these educational practices mediated by Alnuset useful to construct meaning for equations (inequations) in terms of conditioned equalities (inequalities) between two algebraic expressions? (Mariotti, Maracci, \& Sabena, 2007, p.111)

Such a re-formulation consists in a list of questions articulating the CRQ according to the main elements of the specific theoretical framework chosen by the Team. This is a good example for showing the filter effect of a specific theoretical framework: the explicit reference to mediation and to the mediating role of specific educational practices, for instance, makes evident the reference to Activity Theory.

The second example concerns two reformulations referring to the same DDA, Casyopée, developed by DIDIREM.

\section{Team B (DIDIREM)}

How can the representational potentialities offered by Casyopée for connecting the settings of algebra and geometry and for working with parameters support the learning of functions, through what types of situations and with what role of the teacher? (Mariotti, Maracci, \& Sabena, 2007, p.70)

\section{Team C (UNISI)}

Does the sub-environment Geometric Calculation of Casyopée function as a tool of semiotic mediation for the meaning of function as co-variation? Where the meaning of function as co-variation can be articulated in:

- the meaning of both geometrical and numerical variables,

- the meaning of domain of a variable,

- the meaning of function as co-variation over time of variables (even of different kinds: numerical or geometrical).

Does the sub-environment Geometric Calculation of Casyopée function as a tool of semiotic mediation for the processes characterizing the algebraic modelling of a geometrical situation? Where these processes encompass:

- to recognize geometrical variables,

- to associate numbers (numerical variables) to geometrical variables,

- to associate geometrical variables to numbers (numerical variables),

- to pass from not measurable geometrical objects (e.g. points) to measurable geometrical objects,

- to parametrize (cope with the possibly too high number of geometrical variables),

- to express the relation between numerical variables through formulas." (Mariotti, Maracci, \& Sabena, 2007, p.70)

The two reformulations are quite different, both in length and in the number of details that are present. Not all the key elements of the theoretical framework are evident in the first reformulation. For instance, a vision of representations shaped by Duval's approach in terms of semiotic registers does not appear, though it is mentioned in the comments that accompany the question. In the reference to the "types of situations" some evidence appears of the Theory of didactical situations, and of the fact that according to this theory the minimum unit of analysis is the notion of situation. Completely implicit in the formulation of the question is the 
importance attached to instrumentation processes supported by the instrumental approach and to the institutional context supported by the ATD. However, these considerations are explicit in the comments, leading to hypothesize that DIDIREM researchers chose a short reformulation, because they wanted to maintain the spirit of the initial CRQ.

The second reformulation is articulated in two main questions both explicitly referring to the notion of tool of semiotic mediation, a the key construct of the TSM. Each question concerns a different mathematical meaning that is the objective of the mediation process, each meaning is extensively described through a list of components. Such a detailed list, though not explicitly mentioned, is consistent with the theoretical notion of 'semiotic potential' and it is the product of an epistemological and cognitive analysis that has to be carried out a priori.

As a general remark concerning this process of reformulation we want to stress the fact that each team succeeded in reformulating the CRQ in its own theoretical terms for each experimentation. At a first glance, one could consider this a trivial issue, but in our opinion it is not. In general, it is certainly not true that any theoretical frame can be suitable to address any issue. We wish therefore to stress the importance of this result, which we considered as a first and crucial step in the networking enterprise.

As expected, the contribution of different theoretical frameworks led to quite different reformulations of the CRQ highlighting different foci, and showing the richness offered by the complementary of perspectives. However, the reformulation of the CRQ provided us with the possibility of structuring within a uniform frame the local results coming from the different experiments carried out with reference to different theoretical perspectives. The effort done in the design and in the use of the guidelines, described above, was completed by a new methodological tool for presenting our results. Such a shared format was consistent with the Guidelines, adopting a frame and language that emerged from both the Integrative theoretical framework and the Minimal Theoretical Framework. It consisted of two distinct parts: the Teaching Experiment Synthesis Frame, and the Teaching Experiment Analysis Guidelines. The Teaching Experiment Synthesis Frame was intended to give a common structure for summarizing both the main results and the evidence supporting those results. Short summaries of the teaching experiments were also provided. These explained the context of the information needed to make sense of the other teams' answers to their own Research Questions. The Teaching Experiment Analysis Guidelines provided a common structure to frame the answers to the Research Questions addressed through the teaching experiments and formulated in the Teaching Experiment Profiles. Building up from these answers a general answer to the CRQ was finally attempted (Artigue, 2009c). Certainly, the coordination of the answers and the coordination of the different teaching experiment contributions was not completely satisfactory, nevertheless possible paths emerging constituted an important direction for further investigations that in some cases led to interesting results (Maracci, Cazes, Vandebrouck, \& Mariotti, 2009; 2013).

\subsection{The epistemological profile of the DDAs}

The ReMath project combined the development of six different DDAs and their experimentations, based on a vision of their respective didactical functionalities. The diversity of the ReMath DDAs is partially linked to the differences in theoretical perspectives, but it seems much more related to the mathematical domains, which vary from one DDA to another. Moreover some DDAs only refer to mathematical domains, while others link mathematics to other fields such as geography or physics. All DDAs offer some novelty in terms of representations but the distance of these representations to those usually dealt with in educational systems varies greatly. The possibilities of evolution of the representational systems implemented also vary substantially from one DDA to another. However, there exist 
also similarities. Two of the DDAs focus on elementary algebra, two are situated more at the interface between algebra and analysis, while the last two focus on 3D space and geometry. All DDAs connect different systems of representation in an original way, and many of them link some kind of algebraic symbolism to geometrical concepts. Therefore the wide collection of DDAs form a particularly rich landscape for exploring the needs and possibilities of theoretical integration in that area.

In order to better make sense of this diversity and also for facilitating the communication of ReMath outcomes to a wider audience, in the last stage of ReMath, when the development or extension of the DDAs was completed, it was decided to create an epistemological profile for each of the DDAs. The common structure elaborated for the epistemological profiles was organized into four different dimensions: objects, connections, activities, pedagogical/intervention agenda. For each dimension, the nature of the information to be provided was detailed. We reproduced it below from ReMath Deliverable 18 (Artigue, 2006, p. 30-31).

\section{"Objects}

What are the main objects represented and manipulated by/with the DDA?

\section{Representations:}

For each object, describe how they are represented and manipulated by the user. Please distinguish if relevant:

- Dynamic / Static.

- Mathematical / Computational.

- Direct Manipulation / Menu driven.

- Compatible with the curriculum / Innovative

Can the user (teacher or student) build representations of objects ("half-baked" objects)?

\section{Concepts :}

For each object, please indicate the main associated mathematical concepts and the way the representation deals with.

Indicate whether the concepts are rather strictly mathematical or whether the DDA opens to broader concepts (e.g. in physics, geography...)

\section{Connections}

Please describe how the objects are connected.

If possible describe these connections in terms of semiotic systems or semiotic chains.

A map with commented arrows could also be useful.

\section{Activities}

What kind of activities does the DDA aim at:

- Proof

- Exploration

- Modelling

$-\ldots$

Explain how the objects and connections favour this kind of activities.

Are these activities compatible with existing curricula and practice, or are they innovative?

\section{Pedagogical/intervention agenda}

Situate the DDA's design with respect to the following dimensions in terms of pedagogical versus intervention agenda:

1. Innovation / Acceptance

2. Distance to traditional curriculum

3. Status of representations: traditional, innovative, to be handled, related to others, etc.

4. Scope for mathematization or only about mathematics".

Short syntheses of these epistemological profiles can be found in the introduction to this special issue. Regarding, theoretical aspects, the analysis of these profiles reported by Artigue 
(2009c) clearly shows that, while each team of designers was more sensible to one or another theoretical framework, the design decisions, especially regarding representation and manipulation, also depended on an epistemological view of the notions at stake, as well as on pragmatic considerations. The specific theoretical background of each design team was visible in the characteristics of its own DDA. For instance, in the DDAs developed by "constructionist" teams, the compatibility with the curriculum did not seem a major concern, but particular attention was given to offering the learner the possibility of building representations or making them evolve; in the DDA developed in reference to Peirce's semiotics, the design was influenced by the distinction made in the theory between deictic, iconic and symbolic signs. However, the fact that the different teams shared common epistemological views regarding the mathematical objects represented, as pointed out in section 2, and the importance of approaching mathematical concepts through a diversity of representations provided a common ground on which the different teams understood each other, making cross-experiments possible.

\section{Research outcomes in terms of networking}

Within the limits of such an article, some choices need to be made regarding the report on the outcomes of the networking activity carried out in ReMath. Coming back to the hierarchy of strategies mentioned in part I and in the initial landscape presented in part 2, we decided to select outcomes that would illustrate the diversity of forms that productive networking between theories can take. In 4.1 we show how the ReMath activities around these DDAs allowed us to identify both a potential for interesting networking between theories, but also some limits to the integrative enterprise between the Theory of didactical situations (TDS) and the Theory of semiotic mediation (TSM) the one hand, and to the Constructionism and the TDS on the other hand. In 4.2, we show how the ReMath activities made clear that a boundary object (in that case the idea of instrument) may be understood quite differently in different theoretical cultures and how these different understandings may impact the design and use of digital artefacts. In 4.3, finally, we present and discuss what can be seen as the most integrative part of the networking activity: the formulation of shared positions regarding the semiotic affordance of digital technology and conditions for their actualization. This part of the article mainly relies on ReMath Deliverable 18 (Artigue, 2009c).

\subsection{Identifying possible connections and complementarities}

\section{Networking the Theory of didactical situations and the Theory of semiotic mediation}

Some networking between the TDS and the TSM had been already achieved before the launching of ReMath, in Falcade's doctoral thesis (Falcade, 2006), showing that the concept of didactical contract (Brousseau, 1997) was an interesting object for evidencing complementarities between the two theories. However, even if the combined use of these two theories was at the base of the thesis, networking was not its focus. The ReMath crossexperimentation of the Casyopée DDA by the UNISI and DIDIREM teams offered new possibilities for deepening the potential of theoretical connections between the TDS and the TSM, by making these an explicit object of study. Moreover the work was pursued beyond the official end of the ReMath project (Maracci \& al., 2009, 2013). In this paragraph, we synthesize its main results. The TSM and the TDS share the view that tasks lead the learning process, and specifically that tasks can be purposefully designed to lead it. In task design, both the theoretical approaches are especially sensitive to epistemological and mathematical issues. Conceiving learning processes as a combination of adaptation and acculturation, they also pay essential attention to the social organization of task solving and to social interactions in the classroom. These proximities were evident in the distribution of hierarchy between concerns for the two teams. Nevertheless, the TSM and the TDS also present profound 
differences which clearly are reflected in the ways Casyopée's didactical functionalities were viewed by the two teams, in the respective scenarios built for the cross-experimentation, and in the ways the data collected were analyzed and interpreted. The TDS perspective for instance leads to focus on the design of tasks which a priori would make it possible for the mathematical knowledge aimed at to emerge as an optimal solution to the tasks proposed, through the students' adidactic interaction with the milieu of the situation. This implies minimal intervention from the teacher once the students have accepted the mathematical responsibility of solving the task (devolution process), and is in line with the importance attached to the adaptation dimension of learning. The acculturation dimension of the learning process is more specifically taken in charge by institutionalization, a process under the teacher's responsibility. Its purpose is to link the knowledge built by students in the specific context of the task to the institutional knowledge aimed at, both in its content and form, so as to contribute to its de-contextualization. Of course, this is a theoretical model. In the real world, even the realization of well-designed situations with strong adidactic potential does not exactly conform to this model. However, this model strongly influences the design of tasks, and what researchers try to control and optimize within it. It also influences their analysis of subsequent realizations, since in it they contrast the hypotheses and anticipations made explicit in the a priori analysis of the design phase with the a posteriori analysis of realizations. In this model, a DDA such as Casyopée is viewed as an element of the milieu. Its characteristics influence students' interaction with the milieu through the possibilities for semiotic representation and action on the representations it offers, and through the feedback students receive in response to their actions (for instance, when piloting parameters, they can observe their effect on the graphical representations of functions depending on these parameters; when selecting candidates for independent and dependent variables in functional modelling, they receive feedback telling them that either their choice does not work, or that it works; in that case, Casyopée also gives them an algebraic expression for this functional model).

Adopting a TSM perspective means to recognize that this vision of the artefact in terms of action/feedback is too limited, and to acknowledge that artefacts play an essential role in the semiotic activity consubstantial to learning. Accordingly, within a TSM perspective, one should systematically investigate the semiotic activities which occur in the classroom. These take place in collective phases but also in phases of autonomous work when students interact for explaining to each other their respective solutions, for comparing them, or for producing a shared solution. In these occasions students are led to produce signs referring to the solution of the task, hence referring to their activity with the artefact and to the artefact's use. These semiotic activities lead the individuals to making explicit the new knowledge they are developing, which can be related both to the artefact's actual use and to the mathematical knowledge at stake. The analysis focuses on the signs produced by the students referring to the accomplishment of the task and to the use of the artefact, and on how signs are transformed through social activities, which makes a substantial difference with respect to the TDS's focus.

Differences between the TDS and the TSM also affect the vision of the role of the teacher, the way collective phases are organized and managed, and the respective influence given to these with respect to the phases of students' more autonomous work in design. In TDS design, collective phases are generally short, under the responsibility of the teacher and devoted to institutionalization as described above. In TSM design, we see a similar sensitivity to the necessity of institutionalization and decontextualization. But there are also important differences. The semiotic perspective of the TSM shapes the way the connection between the students' personal meanings and mathematical meanings is envisaged. Particular importance is attached to the progressive transformation of signs and their decontextualization both from 
the task context and from the artefact, through social interaction within the classroom. This results in collective phases longer than those observed in TDS designs, much more delicate in their management, and complex in the way they intertwine students' and teacher's contributions. These cannot be reduced to institutionalization phases. This is attested by the fact that a TSM analysis of such phases focuses on the signs produced by teachers and students, and how these signs are transformed through social interactions, and relies, as shown in the Casyopée cross-analysis, on specific constructs such as the idea of semiotic chain ${ }^{4}$, which do not have equivalent in TDS.

Casyopée's cross-analysis, which focused on the collective phases in the two experimentations, showed that these theoretical differences had important and unanticipated consequences. Indeed it made clear that in the DIDIREM design strong attention had been given, as is often the case in TDS design for time constraints, to situations of action, and much less to situations of formulation and validation. Of course, as pointed out in the analysis, several tasks asked students to reflect on their action and to initiate a formulation process, but they did not obey the requirements imposed to situations of formulation, which are especially designed for ensuring a progression in the functionality of mathematics knowledge from a tool for productive action to a tool for efficient expression and communication. This resulted in classroom dynamics where situations of action were directly followed by institutionalization phases where the teacher tried to make collectively emerge from students' adidactic experience the objective mathematical knowledge. The condensation of formulation, validation and institutionalization processes that such an organization induces was an evident source of difficulties which for instance resulted in Topaze effects (Brousseau, 1997): a question posed to students is progressively rephrased up to automatically generating the expected answer. The cross-analysis made it possible to contrast these institutionalization phases with the collective phases in the UNISI experimentation. A first analysis of these phases by DIDIREM researchers also identified Topaze effects in that case, but a second analysis jointly performed by the DIDIREM and UNISI researchers, using the tools provided by the TSM and especially the notion of semiotic chain, convinced researchers that such an interpretation was not appropriate for the Italian collective phases (Maracci \& al., 2013). It is interesting to notice that, in the first analysis, episodes in the transcripts had been determined by looking at changes in the questions at stake or in the distribution of responsibilities between teacher and students (that is to say in accordance with a transcript analysis informed by TDS). In the second analysis, episodes were determined by the beginning and end of semiotic chains. Since along a semiotic chain, responsibility moves between teacher and students, the beginning and ends of the episodes were different; a quite different picture emerged, which invalidated the first interpretation. Beyond that point, the cross-analysis also helped clarify how many designs inspired by the TDS but giving too much importance to situations of actions with respect to situations of formulation, deprived the design from the essential constructs provided by the TDS for copying with semiotic issues, making Topaze effects more likely to happen in the associated realizations.

The results obtained thus confirmed the possibility of local integration of the TDS and the TSM constructs in didactical design that Falcade's thesis had led to hypothesize. In particular, they made clear that the notions of adidactic situation and milieu, which are fundamental constructs in TDS, the ideas of didactic variable and a priori analysis which are fundamental in didactical engineering (Artigue, 2013) could be operational notions for a design activity supported by the TSM. Conversely, they also showed that the vision of semiotic mediation

\footnotetext{
${ }^{4}$ A semiotic chain is meant as a chain of signification connecting highly contextualized signs, strictly related to the use of the artifact, to the mathematical signs that are objects of the teaching-learning activity (Bartolini-Bussi \& Mariotti, 2008, p.756).
} 
and the associated constructs, carried by the TSM, if appropriately integrated in the design could increase the design strength of the TDS, and limit the risks linked to paradoxes of the didactic contract such as the Topaze effect in actual realizations, providing powerful tools for ameliorating the design and the management of the collective phase of Institutionalization and stressing the importance of installing an appropriate didactic contract in the classroom for the success of the didactical engineering hinged upon the process of semiotic mediation. All this makes the concept of didactical contract an interesting object for evidencing complementarities between the two theories (Maracci \& al., 2013).

\section{Networking the TDS and Constructionism:}

In ReMath, networking between the TDS and constructionism could not take so advanced forms as did the networking between the TDS and the TSM, in spite of the evident common base provided by a socio-constructivist approach. Socio-constructivist visions of learning are indeed in the background of the two theories; they are fundamental in constructionism and attached to the adaptation dimension of learning in the TDS. However, the crossexperimentation and cross-analysis made clear that the vision of design supported by the TDS and by constructionism were quite different. As explained above, TDS design is based on a vision of controlled design.

There is no doubt that Constructionist perspectives do not convey a similar vision of design. The design of both the DDAs realized by the Greek team ETL (MaLT and Cruislet), their analysis of didactical functionalities for the given DDAs and the tasks they designed for the cross-experimentation process, provide a coherent picture. What is to be ensured is that the environment provides sufficient opportunities for meaning-making processes. In the design of DDAs, for instance, ETL privileges the vision of digital artefacts as half-baked microworlds that is to say microworlds that the users (teacher or student) can make evolve, adapting them to their own specific needs. It is expected that this adaptation (according to an Instrumental approach, this could be referred to as an instrumentalization process) will generate interesting mathematizations and meaning-making processes. Similarly, in the design of tasks for crossexperimentations, ETL privileges open-ended tasks offering rich potential for meaningmaking processes, but it does not pay much attention to controlling the learning trajectories.

In our opinion, these essential differences make difficult networking that would reach the stage of local integration between the TDS and Constructionism, but it is important to point out that the understanding of these differences and of their impact on design was a result of the ReMath activity and in particular of the cross experimentation method. It must also be noted that, as analyzed in the first article of this special issue, the distance between Cruislet and the digital tools usually used in the French context made problematic the ambition of controlling a design piloted by the TDS for the first experiment carried out by the DIDIREM researchers. It was only for the second experimentation, thanks to the experience gained through the first experimentation and a better knowledge of the tasks developed by the ETL colleagues and understanding of their learning potential, that the DIDIREM team was able to elaborate a controlled scenario adapted to the French students and to exploit the innovative semiotic potential offered by Cruislet. There is no doubt that, when conditions are met, TDS design makes possible a high level of theoretical control over didactical realizations and the associated phenomena, but the ReMath cross-experimentation also showed that, when didactical choices and anticipations are made difficult by a variety of factors, such a design can lead to implementations where the forced realization of anticipated trajectories becomes an obstacle to genuine meaning-making activity. 


\subsection{Making distinctions: the different conceptions and operationalisations of the instrumental approach}

As was mentioned in part 2, all the teams involved in the ReMath project had some knowledge of the research work developed by Rabardel and Vérillon around instrumental issues in the field of ergonomy. Thus since the beginning the distinction between artefact and instrument and the associated notions of instrumental genesis, instrumentalisation and instrumentation were candidates for the status of boundary objects as defined in section 2 . However, participation in the ReMath project helped us to clarify the exact meaning and role given to these notions by the different teams. In this paragraph, we present the results obtained along this dimension of inquiry, discussing separately the case of each of three teams: DIDIREM, UNISI and ETL .

DIDIREM researchers were especially sensitive to instrumental issues. Their work in that area, initiated in the mid nineties around the integration of CAS technology, had played a major role in the development of the Instrumental approach and the design of Casyopée was informed by this approach. Within this approach, as explained in section 2, instrumental issues are embedded in the anthropological perspective offered by ATD, and shaped by the praxeological vision inherent to this theory. More precisely, this leads researchers to think in terms of instrumented praxeologies, that is to say a model of practices structured around types of tasks, instrumented techniques for solving these types of tasks with the support of an artefact, and a theoretical discourse mixing mathematical and instrumental elements making it possible to explain, justify and adapt or generalize these techniques. Therefore, according to this approach, mathematical knowledge resulting from instrumental genesis necessarily intertwines mathematical and instrumental ingredients, and these cannot be artificially separated. Integrating technology into an educational system means accepting this reality, not just introducing technologies for teaching the same mathematics more efficiently with the support of an artefact, and with more resources. It means accepting to give an institutional status to instrumented techniques and to organize their relationships with paper and pencil praxeologies. This has important consequences on design that were visible in the scenarios built by the DIDIREM team. Instrumentalization was progressively organized all throughout the experimentation with tasks mobilizing progressively more and more applications and commands of the DDA. Institutionalization took in charge not only paper and pencil techniques but also instrumented techniques, and paid particular attention to the combination between mathematical and instrumental knowledge underlying these.

The TSM perspective on instrumentation is sensibly different. Artefacts are mainly seen as tools for semiotic mediation. Signs emerge through the activity with artefacts but what is aimed at is an evolution progressively detaching signs from their artefactual reference in order to arrive at conventional mathematical signs. Thus, even if attention is paid to instrumental genesis, it is also expected that what is learnt has to survive the absence of artefacts. Design is thus also impacted but in a different way. In the UNISI scenario, the first session focuses on instrumentalization while the other sessions are organized around the solving of mathematical tasks. The associated collective discussions take place without access to the DDA and this is a deliberate choice. It aims at supporting the double de-contextualization process aimed at, concerning both the particular context of the task and the means for solving it. Students are asked to reflect on what they can draw from this experience for solving a class of tasks with and without the DDA. The fact of having the collective discussion in a place where Casyopée is not available is expected to facilitate the de-contextualization process and the semiotic activity, making it impossible to replay action, obliging to evoke such activity through discourse and gestures. 
The constructionist approach adopted by the Greek and the UK teams leads them to a similar perspective on instrumentation, focusing on instrumentalization. These two teams developed three DDAs (MoPIX, MaLT and Cruislet) which offer an opportunity for evolution through programming in Logo for Malt and Cruislet, and through equations allocating properties and behaviours to physical objects in MoPIX. As explained in the Cruislet epistemological profile, through the use of the Logo programming language the software becomes constructionist in the sense that avatar trips can become parametric models of sequences of displacements. Procedures constituting half-baked microworlds can be designed in the sense that students can observe the trips they define and then change the procedures to make changes to the trips. Furthermore, procedures addressing more than one avatar can be defined making it possible to construct functional relationships between sequences of displacements of pairs of avatars. One such half-baked microworld was the "Guess my flight" game where the user tries to guess the functional relationship either between two planes or between the input and the actual displacement of the plane. In this case, once again, design is influenced by the instrumental perspective, but the scenarios are very different from those produced by the teams which do not rely on Constructionism, because in this case the design exploits the potential of instrumentalization of the artefact in terms of the diversity of the utilizations schemes devised by the students.

ReMath thus helped us understand up to what extent the common reference to the same theoretical approach, here Rabardel's ergonomic approach of instrumental issues, could take different forms according to the theoretical culture it had to be integrated into, and to measure the consequences this could have on the choices made by the DDA designers, on the identification of didactical potentialities of a given technology, on the design of scenarios of use and on the analysis and evaluation of their learning potential.

The notion of Instrumental Genesis, as the combination of instrumentation and instrumentalization, was an obvious boundary object. Less obviously, and despite their strong connection with one particular theoretical framework, at the end of the ReMath project, some other candidate boundary objects emerged, such has the idea of half-baked microworld mentioned above, the idea of adidactic milieu and that of a priori analysis. Regarding this last one, collaboration within ReMath led all the teams to acknowledge the interest of some form of a priori analysis for task design, whatever the theoretical framework inspiring it might be. But it was also acknowledged that a priori analysis, which constitutes a reference for looking at actual realizations of the teaching interventions, necessarily takes on different forms, according to the different theoretical frameworks, in particular with respect to the control on learning trajectories.

\subsection{Towards a consensus regarding semiotic representations}

As explained in part 3, one of the ambitions of the ReMath project was to progressively build consensus about our respective visions of semiotic representations, and the affordances of digital technologies regarding these, transcending our different cultures and theoretical approaches. The first form of consensus achieved, called minimal shared theoretical frame, was rather limited and fully compatible with the different semiotic approaches referred to in the context of the ReMath project. It essentially acknowledged that mathematical objects are only accessible through representations, and that representations must be thought about in triadic terms. Such a minimal shared theoretical frame progressively developed, along the development of the ReMath project, as we became more and more aware of it, and was eventually expressed as a coherent and limited set of positions to which we recognize a deep influence on our vision of the potential of digital artefacts for mathematical learning, and a major role in our identification of their didactical functionalities. Taking into account the demands of the European Commission, these consensus positions were expressed in simple 
terms avoiding technical terms and reference to any particular theoretical approach as much as possible, and they were also oriented towards practical action. We list them below as they appear in ReMath Deliverable 18 (Artigue, 2009c, p. 61-62). They do not have anything spectacular, which is not surprising considering that they constitute in some sense the greatest common divisor that emerged from design work inspired by a substantial diversity of theoretical frameworks.

Position 1: Mathematical objects are not objects directly accessible to our senses. For accessing them, making sense of them, working on them and communicating with others about them, we use external representations of these objects. We also build internal representations that allow us to access them mentally.

Position 2: It is important to consider representations of mathematical objects in a triadic perspective. A representation is something which stands for something else from someone's point of view. It is also important to acknowledge the social and cultural dimension of representations.

Position 3: The notion of semiotic register of representation is a useful notion for approaching the diversity of systems which have been developed for representing mathematical objects, the rules governing the formation and transformation of representations within such systems, and the crucial role played by connections between semiotic registers in mathematical learning.

Position 4: Meaning-making of mathematical objects and processes is a complex semiotic activity mobilizing a diversity of semiotic systems and semiotic activities. An analysis in terms of semiotic registers of representations pays justice to this diversity only partially. The idea of multimodality is a useful idea for approaching the complexity of semiotic systems and activities, thus for understanding the semiotic potential of digital artefacts.

Position 5: Representations of mathematical objects have an essential cultural dimension. Meaningmaking of mathematical objects and processes through these representations is essentially a social activity. The idea of semiotic chain is a useful idea for approaching the processes of evolution of meanings within the classroom discourse, and understanding the role of mediation tools potentially played by DDAs.

Position 6: For understanding the teaching and learning potential of a DDA or in other words for identifying its didactical functionalities, it is important to identify its potential in terms of representation i.e. analyse the DDA's features in terms of representations. This includes its affordances in terms of diversity of representations and ways of dealing with these, in terms of connections between representations, in terms of evolution of representations, and in terms of potential offered to social interactions.

Position 7: When considering the potential of a DDA in terms of representation, it is important to consider that a distinction must be made between didactical functionalities as thought about by the designers of the DDA and those resulting from the interpretations, elaborations and even transformations made by the DDA users.

Position 8: When considering the didactical functionalities of a DDA in a specific educational context, it is important to evaluate the distance in terms of representations between the DDA and the educational context at stake.

Position 9: General perspectives on representations can only partially support the identification of didactical functionalities for a given DDA and mathematical domain. Specific epistemological and didactical perspectives and knowledge concerning the domain at stake are also required.

\section{Discussion and conclusion}

In this article, we have presented the way theoretical networking has been approached in ReMath and some outcomes of this networking. These outcomes are two-fold. On one hand, as shown in section 4, we have obtained practical results in terms of networking situated at different levels of the hierarchic scale presented in section 1, from comparison up to local integration in a few cases; we have also identified limitations of such networking especially 
when a design perspective is adopted, and coherent design choices must be made. On the other hand, through a succession of trials and approximations, we have progressively developed a series of methodological constructs and tools to be used to make such a networking occur, as shown in section 3. In this last section, we would like to reflect on the whole enterprise, pointing out some of its characteristics that, retrospectively, we find especially important, and drawing some general implications for future research work in this rather new area.

A first characteristic is that this enterprise was not the work of a single researcher or of teams sharing the same culture; it was based on the organized interaction of researchers from different cultures. This situation contrasts with the conditions in which networking activities have often been carried out in the past. As made clear along the project, this was an important characteristic for ensuring that the values and constructs of each theoretical framework were correctly understood and used faithfully. Despite our maturity as researchers, we all discovered up to what point our knowledge of many of the theoretical frameworks involved in the project was superficial. We got acquainted with these theoretical frameworks through reading articles, listening to presentations, and discussing with colleagues, but we lacked the first hand experience provided by their actual use in a research project. In such conditions, misunderstanding and distortions were frequent. Using the language of the TDS, we can say that through ReMath, we have created an antagonist milieu for preventing such distortions in networking activities. The existence of experts of the different theoretical approaches involved, and the way their collaboration was organized were essential to the creation of such an antagonist milieu.

A second characteristic is that the possibilities of networking were not examined exclusively in terms of potential for enriching some form of a posteriori analysis, which is often the case in networking activities; it was also examined in terms of potential for guiding design, both the design of digital artefacts and task design, which is much more demanding. The crossexperimentation of Cruislet piloted by the ETL team in Greece and the DIDIREM team in France, was especially insightful from this perspective. Despite sharing a socio-constructivist background, it became clear for the two teams that it was impossible to combine the respective design perspectives of Constructionism and that of the TDS. However, it must be pointed that the ETL and the DIDIREM researchers could fruitfully interact, comparing and contrasting their design principles, and enriching their respective a posteriori analyses through these interactions. Moreover, as a result of this networking process, the DIDIREM team was able to adapt some of the tasks developed by the ETL team in a further experimentation of Cruislet, something that had seemed impossible initially. Such advances are especially important considering that design in mathematics education lies at the interface between theory and practice.

A third characteristic directly results from the vision adopted in ReMath regarding theories as structured systems emerging from research practice and conditioning all facets of these practices. Taking seriously into account this vision imposes that networking methodologies allows researchers to access and work on the different facets of these practices, paying the necessary attention to the problems of visibility and naturalization, as explained in section 2 . This was effectively done in ReMath through a purposeful, complex and progressive construction of methodological tools which has been reported in section 3. This is in line with the perspective developed by Artigue, Bosch, and Gascón (2011) in terms of research praxeology, thus we want to spend a few words commenting on it.

At the time of ReMath, the idea of research praxeology did not exist, but today, it appears a good vehicle for going further in this retrospective analysis. It starts from the idea that envisaging networking at the sole level of theories is a limited view as theories are not 
isolated entities. They are ingredients of the research practices that they shape, and are shaped by them. According to the ATD perspective, research practices, as any kind of human practice, may be modelled in terms of praxeologies (see 4.3). Hence, to be effective, a networking enterprise should focus not solely on theories, rather on research praxeologies made of research questions and tasks, research techniques for addressing these questions or tasks, the methodology developed for explaining and justifying these techniques, as well the theoretical discourse underpinning it. Looking back at the ReMath enterprise, this is clearly what was done, considering research praxeologies oriented towards design of digital artefacts and design of scenarios of use. As a matter of fact, in ReMath networking, one can observe two levels of research praxeologies. At the first level, there are research praxeologies proper to each team and reflecting its research culture; at the second level, there are original research praxeologies, built on top of the first ones, and aimed at supporting the networking process, what we will call here 'networking praxeologies'. These networking praxeologies are organized around a series of tasks and tools designed to support the accomplishment of these tasks: answering questionnaires, reformulating questions, carrying-out cross analyses... As shown above, solving these tasks requires the development of techniques, partly original, for organizing the design of the experiments, the collection of data and the productive interaction of the different partners. The novelty of this area of research makes the practical block of networking praxeologies, both tasks and techniques, not yet well established; they are rather art-craft objects whose potential is tested in action, and which must be progressively refined. In our opinion, the ReMath experience has substantially contributed to the development and the enrichment of this practical block. ReMath has also shown that it is not easy at all to make research praxeologies a shared object of study without denaturing them. For instance, a technique based on common research questions as used here strongly limits the expression of the richness and depth of the research praxeologies of a given culture, even in relation to a very specific topic. This was the reason why it was decided in ReMath that each team would be offered the possibility of complementing the common research question with more specific questions. Thanks to this decision, in effect for both the two teams involved, each crossexperimentation was expected to be a good representative of each team's own research praxeologies. At the same time, cross-experimentation methodology was of course part of a networking praxeology, providing techniques for answering research questions related to the networking process.

The theoretical block of networking praxeologies is also in the process of emerging, perhaps, at he moment it is in a less developed state. If one excepts the categories which have been identified by Prediger, Bikner-Ahsbahs, and Arzarello (2008), the theoretical discourse on networking is nearly nonexistent. Up to what point has ReMath contributed to this theoretical block? In our opinion, the methodological discourse developed through the project in connection with the specific techniques developed and the constructs supporting this methodological discourse offer some ingredients for the elaboration of this theoretical block. We see this to be especially the case for the meta-language of concerns, and also of the boundary objects identified, but much more remains to be done in order to develop a well organized discourse around these elements. In the paper by Artigue, Bosch, \& Gascón (2011) it is stressed that research praxeologies are dynamic entities and that didactical phenomena play a crucial role in these dynamics. Some potentially interesting phenomena have certainly emerged from ReMath, for instance the different interpretations of Rabardel's ideas according to the theoretical frameworks they were embedded in. We conjecture that here we touch an occurrence of a more general phenomenon, especially important when approaching networking issues, but the understanding we have of it and of the mechanisms underlying it, once again, calls for further research. 
Looking retrospectively at the project ReMath and at its outcomes, with the support of the idea of research praxeologies, helps us to identify some of its characteristics that contributed to its success, and to identify how it might contribute to the emergence of a new kind of research praxeologies: the networking praxeologies, which can be situated at a meta-level with respect to ordinary research praxeologies. Looking at the concluded project also helps understand its limitations and the fact that we are still at the beginning of a long journey with respect to networking.

\section{References}

Artigue, M. (2002). Learning mathematics in a CAS environment: The genesis of a reflection about instrumentation and the dialectics between technical and conceptual work. International Journal of Computers for Mathematical Learning, 7, 245-274.

Artigue, M. (coord.) (2006). Integrative theoretical framework. ReMath Deliverable 1. IST426751. http:// www.remath.cti.gr

Artigue, M. (Ed.) (2009a). Connecting Approaches to Technology Enhanced Learning in Mathematics: The TELMA Experience. International Journal of Computers for Mathematical Learning, 14(3).

Artigue M. (2009b). Rapports et articulations entre cadres théoriques : le cas de la théorie anthropologique du didactique [Relations and articulations between theoretical frameworks: the case of the anthropological theory of didactics]. Recherches en Didactique des Mathématiques, 29/3, 305-334.

Artigue, M. (coord.) (2009c). Integrative Theoretical Framework - Version C. ReMath Deliverable 18. IST4-26751. http:// www.remath.cti.gr

Artigue, M. (2013). Didactic engineering in mathematics education. In, S. Lerman (Ed.) Encyclopedia of Mathematics Education: Springer Reference (www.springerreference.com). Berlin - Heidelberg: Springer-Verlag.

Artigue M., Bosch, M., \& Gascón J. (2011). Research praxeologies and networking theories. In, M. Pytlak, T. Rowlad, \& E. Swoboda (Eds). Proceedings of the Seventh Congress of the European Society for Research in Mathematics Education (pp. 281-290). Rzeszów: University of Rzeszów.

Bartolini Bussi, M.G. \& Mariotti, M.A. (2008). Semiotic mediation in the mathematics classroom: artifacts and signs after a Vygotskian perspective. In, L.English, M. Bartolini Bussi, G. Jones, R. Lesh, \& D. Tirosh (eds.) Handbook of International Research in Mathematics Education, second revised edition, (pp. 746-805). Mahwah, NJ: Lawrence Erlbaum.

Bikner-Ahsbahs, A., Dreyfus, T., Kidron, I, Arzarello, F., Radford, L., Artigue, M., \& Sabena, C. (2010). Networking of theories in mathematics education. In, Pinto, M.M.F. \& Kawasaki, T.F. (Eds.) Proceedings of the $34^{\text {th }}$ Conference of the International Group for the Psychology of Mathematics Education (Vol. 1, pp. 145-175). Belo Horizonte, Brazil.

Brousseau, G. (1997) Theory of didactical situations in mathematics. Dordrecht: Kluwer Academic Publishers.

Chevallard, Y. (1992), Concepts fondamentaux de la didactique. Perspectives apportées par une approche anthropologique. [Fundamental concepts of didactics. Perspectives provided by an anthropological approach]. Recherche en didactique des Mathématiques 12(1), 73-112. 
Chevallard, Y. \& Sensevy, G. (2013). Anthropological Approaches in Mathematics Education, French Perspectives. In: Lerman S. (Ed.) Encyclopedia of Mathematics Education: springerreference (www.springerreference.com). Berlin - Heidelberg: Springer-Verlag

Duval, R. (1995). Sémiosis et pensée humaine. Bern: Peter Lang.

Engeström, Y. (1999). Activity theory and individual and social transformation. In Y. Engeström,R. Miettinen,R-L. Punamäki (Eds) Perspectives on activity theory, (pp. 19-38). Cambridge: Cambridge University Press.

Falcade, R (2006) Théorie des Situations, médiation sémiotique et discussions collectives dans des séquences d'enseignement qui utilisent Cabri-géomètre et qui visent à l'apprentissage des notions de fonction et graphe de fonction. [Theory of didactic situations, semiotic mediation and collective discussions in teaching sequences involving the use of Cabri-géomètre and aiming at the learning of function and graphs]. Thèse de doctorat. Université de Grenoble 1.

Halliday, M. A. K. (1978). Language as social semiotic: the social interpretation of language and meaning. London: Edward Arnold.

Harel, G., \& Papert, S. (Eds.). (1991). Constructionism. Norwood, NJ: Ablex.

Kafai, Y., \& Resnick, M. (Eds.). (1996). Constructionism in Practice: Designing, thinking and learning in a digital world. Mahwah, NJ: Lawrence Erlbaum Associates.

Lagrange, J.B. (2000). L'intégration d'instruments informatiques dans l'enseignement : une approche par les techniques. [The integration of computer tools in education: an approach through techniques]. Educational Studies in Mathematics, 43(1), 1-30.

Lave, J., Wenger, E. (1991). Situated learning: legitimate peripheral participation. New York: Cambridge University Press.

Leont'ev, A. N. (1978). Activity, consciousness and personality. Englewood Cliffs: PrenticeHall.

Mariotti M.A. \& Maracci M. (coords) (2007) Design-based research: process and results ReMath Deliverable 13. IST4-26751. http:// www.remath.cti.gr

Mariotti M.A. Maracci M. \& Saberna (coords) (2007) Research design ReMath Deliverable 11. IST4-26751. http:// www.remath.cti.gr

Maracci M., Cazes, C., Vandebrouck, F., \& Mariotti, M.A. (2009); Casyopée in the classroom: two different theory-driven pedagogical approaches. In V. Durand-Guerrier, S. Soury-Lavergne \& F. Arzarello (Eds) Proceedings of the 6th Conference of the European Society for Research in Mathematics Education. (pp. 1399-1408). Lyon: Service des publications, INRP.

Maracci, M., Cazes, C., Vandebrouck, F., \& Mariotti, M. A. (2013) Synergies between theoretical approaches to mathematics education with technology: a case study through a cross-analysis methodology, Educational Studies in Mathematics, 84(3), 461-485

Niss, M. (2007). Reflections on the state and trends in research on mathematics teaching and learning: from here to utopia. In, F. Lester (Ed.), Second handbook of research on mathematics teaching and learning, (pp. 1293-1311). Greenwich, Connecticut: Information Age Publishing, Inc.

Peirce, C.S. (1931/1958). Collected Papers. Cambridge M.A.: Harvard University Press. 
Prediger, S., Bikner-Ahsbahs, A., \& Arzarello, F. (2008). Networking strategies and methods for connecting theoretical approaches: first steps towards a conceptual framework. ZDM - The International Journal on Mathematics Education, 40(2), 165-178.

Rabardel, P. (1995): Les hommes and les technologies. Approche cognitive des instruments contemporains.[Technologies and human beings. A cognitive approach to contemporary instruments]. Paris: A. Colin.

Radford, L. (2008). Connecting theories in mathematics education: challenges and possibilities. ZDM - The International Journal on Mathematics Education, 40(2), 317-327.

Silver, E.A. \& Herbst, P. (2007). Theory in Mathematics Education Scholarship. In, F. Lester (Ed.), Second Handbook of Research on Mathematics Teaching and Learning, 39-67. Greenwich, Connecticut: Information Age Publishing, Inc. .

Sriraman, B. \& English, L. (Eds.) (2010). Theories of Mathematics Education. Seeking New Frontiers. New York: Springer.

Star, S.L. \& Griesemer, J.R. (1989) Institutional ecology, "translations" and boundary objects: amateurs and professionals in Berkeley's museum of vertebrate zoology, 1907 -39, Social Studies of Science, 19(3), 387-420.

TELMA ERT (2006). Developing a joint methodology for comparing the influence of different theoretical frameworks in technology enhanced learning in mathematics: the TELMA approach. In Le Hung Son, N. Sinclair, J.-B. Lagrange \& C. Hoyles (eds.) Proceedings of the ICMI 17 Study Conference: background papers for the ICMI 17 Study. (2, pp. 46-55). Hanoï : Hanoï University of Technology.

Vérillon, P \& Rabardel, P. (1995). Cognition and artifacts : a contribution to the study of thought in relation to instrumented activity, European Journal of Psychology of Education, 10(1),77-101

Vygotsky, L. S. (1978). Mind in society. The development of higher psychological processes. Cambridge, MA: Harvard University Press. 


\section{Appendix 1: Elements of the first ITF: Key concerns and didactical functionalities}

\begin{tabular}{|c|}
\hline Integrative Theoretical Framework \\
\hline $\begin{array}{l}\text { Part } 1 \text { : Contextual characteristics of the project under study } \\
\text { How are the following dimensions of context taken into consideration at a theoretical level in the project? What } \\
\text { constructs are used for this purpose? } \\
\text { - The situational context of the project } \\
\text { - The institutional/cultural context of the project }\end{array}$ \\
\hline $\begin{array}{l}\text { Part } 2 \text { : Didactical functionalities and design } \\
\text { For each dimension of didactical functionalities, a list of concerns is given. You are asked to grade them from } 0 \text { to } \\
5 \text {, this grade reflects the level of priority given in design ( } 0 \text { not considered, } 5 \text { high priority). In a second phase, } \\
\text { you are asked to say what are the theoretical frames you use, if any, when taking into account these concerns, and }\end{array}$ \\
\hline
\end{tabular}

\section{a) Characteristics of the ILE (or of the set of ILEs if several ILEs are concerned by design)}

Are the following concerns given a high priority in your design (grade from 0 to 5: 0 not considered, 5 high priority):

- concerns about the ways mathematical objects and their interaction are represented?

- concerns about the ways didactic interactions are represented?

- concerns about the ways representations can be acted on?

- concerns about possible interactions, connections with other semiotic systems, including the representations provided by other DDAs?

- concerns about the relationships with institutional or cultural systems of representation?

- concerns about the rigidity/evolutive characteristics of representations?

For those considered, what are the theoretical frames and constructs, if any, which you refer to:

- at the level of general principles and metaphors?

- at an operational level?

\section{b) Educational goals}

When thinking about educational goals to be associated to the ILE or set of ILEs, in the design phase, what concerns are given a high priority (grade from 0 to 5 ):

- epistemological concerns?

- semiotic concerns?

- cognitive concerns?

- social concerns?

- cultural and institutional concerns?

Up to what point are those considered linked to representational characteristics of the ILE or set of ILEs (grade from 0 to 5: 0 no link, 5 strong link) ?

For those linked, what are the theoretical frames and constructs, if any, used for this linkage:

- at the level of general principles and metaphors?

- at an operational level?

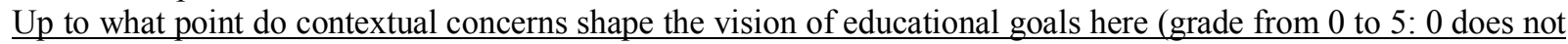
shape, 5 strongly shapes):

- local concerns?

- global concerns?

What are the theoretical frames and constructs, if any, used:

- at the level of general principles and metaphors?

- at an operational level?

\section{c) Modalities of use}

When thinking about possible modalities of use in the design of this ILE or set of ILEs, what concerns were given a high priority (grade from 0 to 5 ):

- $\quad$ concerns about the mathematical tasks and their temporal organization ?

- concerns about the functions to be given to the artefact and their possible evolution?

- concerns about semiotic issues?

- concerns about instrumentation processes?

- concerns about social organization and interactions?

- institutional and cultural concerns?

Up to what point are those considered linked to representational characteristics of the ILE (grade from 0 to 5)?

For those linked, what are the theoretical frames and constructs, if any, used for this linkage

- at the level of general principles and metaphors?

- at an operational level?

Up to what point do contextual concerns shape the vision of modalities of use (grade from 0 to 5): 


\begin{tabular}{|c|}
\hline $\begin{array}{l}\text { - local concerns? } \\
\text { - global concerns? } \\
\text { What are the theoretical frames and constructs, if any, used: } \\
\text { - at the level of general principles and metaphors? } \\
\text { - at an operational level? }\end{array}$ \\
\hline Part 3: Analysis of use \\
\hline $\begin{array}{l}\text { Collection of data } \\
\text { How are concerns about representations and contexts taken into account in the collection of data as regards the } \\
\text { use of ILEs? } \\
\text { What are the theoretical frames and constructs, if any, used for this: } \\
\quad \text { - at the level of general principles and metaphors? } \\
\quad \text { - at an operational level? }\end{array}$ \\
\hline $\begin{array}{l}\text { Analysis of data } \\
\text { How are concerns about representations and contexts taken into account in the analysis of data as regards the use } \\
\text { of ILEs? } \\
\text { What are the theoretical frames and constructs, if any, used for this: } \\
\quad \text { - at the level of general principles and metaphors? } \\
\quad \text { - at an operational level? }\end{array}$ \\
\hline
\end{tabular}

